\title{
ON THE PLANCHEREL THEOREM OF THE $2 \times 2$ REAL UNIMODULAR GROUP
}

\author{
BY LAJOS PUKANSZKY ${ }^{1}$ \\ Communicated by Ralph Phillips, January 22, 1963
}

1. Introduction. Let $G$ be the group of the $2 \times 2$ real unimodular matrices, and $C_{c}^{\infty}$ the family of all indefinitely differentiable functions on $G$ with a compact support. It is known that there exists a family $T$ of equivalence classes of irreducible unitary representations of $G$, the members of which, using the notations of [1], we denote by $C_{l}^{(j)}, D_{l}^{+}$and $D_{l}^{-}$resp. $\left(j=0, \frac{1}{2}, \frac{1}{4}<q<+\infty, l=\frac{1}{2}, 1, \frac{3}{2}, \cdots\right)$ with the following properties. (1) For any representation $U(a)$ in $T$ and any $f \in C_{c}^{\infty}$ the operator $U_{f}=\int_{a} f(a) U(a) d \mu(a)$ is of trace class; here $d \mu(a)$ is the element of a fixed left invariant Haar measure on $G$ (for the normalization to be used cf. below) and the trace, considered as a linear operation on $C_{c}^{\infty}$, is a distribution. (2) Putting $T_{\lambda}^{(+)}(f)\left(T_{\lambda}^{(-)}(f)\right)$ for this distribution if $U(a)$ belongs to the class $C_{a}^{(0)}\left(C_{a}^{(1 / 2)}\right.$ resp., $\left.q=\frac{1}{4}+\lambda^{2}, \lambda>0\right)$, and $T_{l}(f)$ if $U(a)$ is the direct sum of a representation of class $D_{l}^{+}$with a representation of class $D_{l}^{-}$, we have

$$
\begin{aligned}
f(e)= & \int_{0}^{\infty} \tanh \pi \lambda T_{\lambda}^{(+)}(f) d \lambda+\int_{0}^{\infty} \lambda \operatorname{coth} \pi \lambda T_{\lambda}^{(-)}(f) d \lambda \\
& +\sum_{j=1}^{\infty} \frac{j-1}{2} T_{j / 2}(f) .
\end{aligned}
$$

Here $e$ is the unit element of $G$. (1) is called the Plancherel formula for $G$.

Proofs for (1) were outlined by V. A. Bargmann [1, cf. esp. $\$ 13$, p. 638] and Harish-Chandra [4]. The purpose of the present note is to suggest a new approach as a special case of a more general method to be applied later to the investigation, begun in [2], of the discrete series $\left(D_{l}^{ \pm}\right.$for $G$ ) of Lorentz groups of higher dimension. Before giving the details we sketch for later use a slightly modified version of Bargmann's proof. All necessary properties of the representations of $T$ can easily be verified through the realizations described below in $\S 3$.

We consider the subgroup $G_{1}$ of rotations of $G$, and put

1 This work was done during the author's stay at Stanford University. 


$$
g_{\phi}=\left(\begin{array}{rr}
\cos \frac{\phi}{2} & -\sin \frac{\phi}{2} \\
\sin \frac{\phi}{2} & \cos \frac{\phi}{2}
\end{array}\right) \quad(0 \leqq \phi<4 \pi) .
$$

Next we observe that both sides of (1) remain invariant when replacing $f(g) \in C_{c}^{\infty}$ by $f\left(o g o^{-1}\right)$ ( $o \in G$ fixed); it follows easily that it suffices to verify (1) for the subfamily $F$ of $C_{c}^{\infty}$ consisting of functions invariant under this substitution. We denote the collection of elements of $F$ satisfying $f\left(g_{\phi} g\right)=f\left(g g_{\phi}\right)=e^{i n \phi} f(g)$ by $F_{n}\left(n=0, \pm \frac{1}{2}, \pm 1, \pm \frac{3}{2}, \cdots\right)$. Then it is even enough to assume that $f$ lies in $F_{n}$ for some $n$; the case of any $f \in F$ then can be verified by using the linearity of both sides in (1), considering $F$ as direct sum of the $F_{n}$ 's and performing an appropriate limit transition. We now introduce a second subgroup $G_{2}$ of $G$ with the typical element

$$
g_{u}=\left(\begin{array}{cc}
\cosh \frac{u}{2} & \sinh \frac{u}{2} \\
\sinh \frac{u}{2} & \cosh \frac{u}{2}
\end{array}\right) \quad(-\infty<u<+\infty) .
$$

Elements of the form $g_{\phi_{1}} g_{u} g_{\phi_{2}}\left(u \geqq 0,0 \leqq \phi_{j}<4 \pi, j=1,2\right)$ cover the submanifold $G-G_{1}$ exactly twice. Using $\left(\phi_{1}, u, \phi_{2}\right)$ as coordinates, we get an invariant volume element by putting

$$
d \mu(a)=\frac{\sinh u}{(4 \pi)^{2}} d \phi_{1} d u d \phi_{2} .
$$

Suppose now $f \in F_{n}, U(a) \in T$ and consider $\operatorname{Tr}\left(U_{f}\right)$. If we have

$$
U\left(g_{\phi}\right) h=e^{-i m \phi} h \quad(0 \leqq \phi<4 \pi),
$$

then using (2) one sees at once that $\left(U_{f} h, h\right)=0$ if $n \neq m$ and $=\int_{0}^{\infty} f(u)\left(U\left(g_{u}\right) h, h\right) \sinh u d u$ if $n=m$. On the other hand it is known that if $k$ in the representation space is such that $(U(a) k, k)$ is sufficiently regular, then it is an eigenfunction of the Laplacian $L$ of $G$ with an eigenvalue depending on the equivalence class of $U(a)$ only; for $C_{q}^{(0)}$ and $C_{q}^{(1 / 2)}$ this is $q$, for $D_{l}^{ \pm} l(1-l)$. It turns out that any eigenelement $h$ of the restriction of $U(a)$ to $G$ possesses this property. Assuming $\|h\|=1$, we have for the function $F(a)$ $=(U(a) h, h): F\left(g_{\phi} a\right)=F\left(a g_{\phi}\right)=F(a) e^{-i m \phi}, F(e)=1$ and $L F=c F$ with suitable $c$. Using the expression of $L$ by $\phi_{1}, u, \phi_{2}$ 


$$
\begin{aligned}
L=-\left(\frac{1}{(\sinh u)} \frac{\partial}{\partial u}\right. & (\sinh u) \frac{\partial}{\partial u} \\
& \left.+\frac{1}{(\sinh u)^{2}}\left[\frac{\partial^{2}}{\partial \phi_{1}^{2}}-2 \cosh u \frac{\partial^{2}}{\partial \phi_{1} \partial \phi_{2}}+\frac{\partial^{2}}{\partial \phi_{2}^{2}}\right]\right)
\end{aligned}
$$

one immediately sees that $F(a)$ and hence, because of the irreducibility of $U(a)$ (up to a factor of absolute value 1), $h$ is uniquely determined. Observe, incidentally, that $F\left(g_{u}\right)$ is real which implies $F\left(g_{u}\right)$ $=F\left(g_{-u}\right)$. The range of $m$ is the collection of all integers, or half integers in the case of $C_{q}^{(0)}\left(C_{a}^{(1 / 2)}\right.$ resp.), and the set of all integers, or half integers $\geqq(\leqq l)$ in the case of $D_{l}^{+}$( $D_{l}^{-}$resp.) according to whether $l$ is integer or half integer resp. Hence, putting $a_{n}^{(\lambda)}(\cosh u)$ or $a_{n}^{(l)}(\cosh u)$ for $\left(U\left(g_{u}\right) h, h\right)$ if $U(a)$ is of class $C_{g}^{(0)}, C_{q}^{(1 / 2)}\left(q=\frac{1}{4}+\lambda^{2}\right)$ or $D_{l}^{ \pm}$and $U\left(g_{\phi}\right) h \equiv e^{-i n \phi} h,\|h\|=1$ (provided it exists), to prove (1) it suffices to show that if $f(u) \in C^{\infty}, f(u) \equiv f(-u)$ vanishes outside a compact set (in what follows $C_{c}^{\infty}$ will stand for these functions) one has

$$
\begin{aligned}
f(e)= & \int_{0}^{\infty} \tanh \pi \lambda\left[\int_{0}^{\infty} f(u) a_{n}^{(\lambda)}(\cosh u) \sinh u d u\right] d \lambda \\
& +\sum_{l=1}^{n}\left(l-\frac{1}{2}\right) \int_{0}^{\infty} a_{n}^{(l)}(\cosh u) f(u) \sinh u d u
\end{aligned}
$$

if $n$ is integer, and

$$
\begin{aligned}
f(e)= & \int_{0}^{\infty} \operatorname{coth} \pi \lambda\left[\int_{0}^{\infty} f(u) a_{n}^{(\lambda)}(\cosh u) \sinh u d u\right] d \lambda \\
& +\sum_{l=1}^{n+1 / 2}(l-1) \int_{0}^{\infty} a_{n}^{(l-1 / 2)}(\cosh u) f(u) d u
\end{aligned}
$$

if $n$ is half integer.

According to Bargmann, one proves (4) and (4') by showing that the part of the system $\left\{a_{n}^{(\lambda)}(\cosh u), a_{n}^{(l)}(\cosh u)\right\}$ occurring in these formulas gives the eigenfunction expansion of an appropriate selfadjoint extension of the ordinary differential operator $L_{n}$ of the second order defined by

$$
e^{i n\left(\phi_{1}+\phi_{2}\right)}\left(L_{n} f\right)(u)=(L f)\left(g_{\phi_{1}} g_{u} g_{\phi_{2}}\right) \quad\left(f \in F_{n}\right) .
$$

Observe finally that, since the right-hand sides of (4) and $\left(4^{\prime}\right)$ are invariant under the substitution $n \rightarrow-n$, it suffices to consider the case $n \geqq 0$ only.

In the present note we sketch a proof of the formulas (4) and $\left(4^{\prime}\right)$ 
and hence of the Plancherel theorem itself. The method consists in adapting the classical idea of constructing singular integrals through solving boundary value problems (cf. in particular Szegö's reasoning to prove the completeness of the spherical functions in [3, pp. 388$389])$. With a rough analogy our method, when compared with Bargmann's, corresponds to establishing the completeness of the system of trigonometrical functions using Poisson's integral instead of considering it as the system of eigenfunctions of a certain differential operator.

Before stating the precise form of the steps leading to the proof we wish to discuss the idea of the method in some detail.

2. Background of the method. We consider the space of the variables $\left(x_{0}, x_{1}, x_{2}\right)$ with the Lorentzian metric $(d s)^{2}=\left(d x_{0}\right)^{2}-\left(d x_{1}\right)^{2}$ $-\left(d x_{2}\right)^{2}$, and introduce hyperbolic coordinates in the upper half cone $C\left(x_{0}^{2}-x_{1}^{2}-x_{2}^{2}>0, x_{0}>0\right)$ by setting $x_{0}=r \cosh u, x_{1}=r \sinh u \cos \phi$, $x_{2}=r \sinh u \sin \phi(u>0)$. Denoting the wave operator $\partial^{2} / \partial x_{0}^{2}-\partial^{2} / \partial x_{1}^{2}$ $-\partial^{2} / \partial x_{2}^{2}$ by $\Delta$, we form in $C$ the differential operator $D_{n} f=\Delta f+q_{n} f$, where $q_{n}(r, u, \phi) \equiv 2 n^{2} /\left(r^{2}(1+\cosh u)\right)\left(n=0, \frac{1}{2}, 1, \ldots\right)$, and observe immediately that by virtue of (3) and (5) we have

$$
r^{2} D_{n}=\frac{\partial}{\partial r} r^{2} \frac{\partial}{\partial r}+\frac{1}{(\sinh u)^{2}} \frac{\partial^{2}}{\partial \phi^{2}}+L_{n} .
$$

We denote the point with $x_{0}=\rho, x_{1}=x_{2}=0(\rho>0)$ by $P_{\rho}$, and by $C_{\rho}$ the cone $0=\left(x_{0}-\rho\right)^{2}-x_{1}^{2}-x_{2}^{2}$. Consider now the function $E_{n}(p)$, with $p=(r, u, \phi)$ inside $C_{1}$, defined by

$$
E_{n}(p)=r^{n}\left(\frac{1+\mu}{2}\right)^{n} G_{n}(r, \mu)
$$

where $G_{n}(r, \mu)=2^{2 n} / R(1+\rho+R)^{2 n}, R=\left(1-2 \mu r+r^{2}\right)^{1 / 2}, 1-2 \mu r+r^{2}>0$, $\mu=\cosh u . G_{n}$ is just the generator function of the Jacobi polynomials $P_{j}^{[0,2 n]}$ (cf. [5, p. 69]). It turns out that the fundamental solution $E_{n, \rho}(p)$ of $D_{n} f=0$, belonging to the point $P_{\rho}$, is given by $\rho E_{n}(\rho, \mu)$. Let $H$ be the space-like surface consisting of the part of the hyperboloid $x_{0}^{2}-x_{1}^{2}-x_{2}^{2}=1$ in $C$, and $f$ a sufficiently regular function on $H$. The solution of $D_{n} F=0$, vanishing on $H$ and with values prescribed by $f(p)$, for the derivative according to the Lorentzian normal, pointing inwards, to $H$, in points of the $x_{0}$-axis is given by

$$
F\left(P_{\rho}\right)=\int_{H_{\rho}} E_{n, \rho}(p) f(p) d v
$$

where $H_{\rho}$ is the intersection of $H$ and $C_{\rho}$, and $d v$ the Lorentzian surface 
element. Consider now an $f \in C_{c}^{\infty}$ as an axially symmetric function on $H$. Substituting this in (7) expressed in hyperbolic coordinates, using $E_{n, \rho}(1, \mu)=\rho^{2} E_{n}(\rho, \mu)$ and observing that the right derivative according to $r$ at $r=1$ is just the derivative according to the inward Lorentzian normal, we get

$$
c f(0)=\left.\frac{d}{d r} \int_{0}^{\log r} E_{n}(r, \mu) f(u) \sinh u d u\right|_{r=1}
$$

where $c$ does not depend on $f$. Extend now, for a fixed $u \geqq 0$, the definition of $E_{n}(r, \mu)$ for all $r>0$ by setting it equal to 0 for $e^{-u} \leqq r \leqq e^{u}$; assume a formula of the type

$$
E_{n}(r, \mu)=-\sum_{(l)} a_{l}(\mu) r^{-l}+\int_{0}^{\infty} c(\lambda) r^{-1 / 2+i \lambda} a_{\lambda}(\mu) d \lambda
$$

with sufficiently regular $a_{l}(\mu)$ and $a_{\lambda}(\mu)$, assuming 1 for $u=0$; in the first member on the right-hand side $n$ runs over all positive integers (half integers) not exceeding $n$. Then using $D_{n} E_{n}=0$ one could show $L_{n} a_{l}=l(1-l) a, L_{n} a_{\lambda}=q a_{\lambda}\left(q=\frac{1}{4}+\lambda^{2}\right)$. Multiplying finally both sides with $f(u) \sinh u$, integrating, and using (8) purely formally, one gets (4) and $\left(4^{\prime}\right)$. In what follows we show how to derive all this in a precise fashion. Instead of using the operator $D_{n}$ we prefer to identify the functions occurring in (9) by aid of certain realizations of the representations in $T$. In this fashion, incidentally, we obtain a set of integral representations for a class of special functions. We shall discuss the connection of the functions $E_{n}$, through recurrence relations, having simple group theoretic interpretation with the hyperbolic potential (case $n=0$ ), in a subsequent note.

3. Sketch of the proof. Keeping $\mu=\cosh u, u>0$ fixed, we replace $r$ by the complex variable $t$ in the expression of $G_{n}(r, \mu)$ (cf. (6); $n \geqq 0$ integer or half integer). We get a univalued analytic function on the complex plane cut along $\left[e^{-u}, e^{u}\right]$, by taking for $R$ that branch which is $>0$ for $t<e^{-u}$ and $<0$ for $t>e^{u}$. For any fixed complex $z$ by $t^{z}$ we mean $\exp z(\log t)$, where $\log t$ stands for the branch, real for $t>0$, and made univalent through a cut along $[-\infty, 0]$. We define

$$
P_{z}^{[0,2 n]}(\mu)=\frac{1}{2 \pi i} \int_{C} G_{n}(t, \mu) \frac{d t}{t^{z+1}}
$$

where $C$ is any closed curve encircling the segment $\left[e^{-u}, e^{u}\right]$ clockwise. By deforming the path of integration, one sees that for $\operatorname{Re} z>$ $-(2 n+1)$ we also have 


$$
P_{z}^{[0,2 n]}(\mu)=\frac{1}{2 \pi i} \int_{C^{\prime}} G_{n}(t, \mu) \frac{d t}{t^{z+1}}
$$

where $C^{\prime}$ is a curve, oriented counterclockwise, surrounding the interval $[-\infty, 0]$ sufficiently closely. The notation has been chosen in conformity with the standard one for Jacobi polynomials (obtained for positive integral $z$ ). Though not needed in the following we observe that we have $P_{z}^{[0,2 n]}(\mu)=F(-z, 2 n+z+1,1 ;(1-\mu) / 2)$, as one can verify using the differential equation of the hypergeometric functions. Next we put

$$
\frac{1}{R(1+t-R)^{2 n}}-\frac{1}{R(1+t+R)^{2 n}}=\frac{P(t)}{(1+\mu)^{2 n} t^{2 n}} .
$$

It is easy to see, that for $n>0$ the coefficient of $t^{i}$ in the polynomial $P(t)$ of degree $2 n-1$ is just $P_{j}^{[0,-2 n]}(\mu)$; furthermore we have $P(t)$ $\equiv t^{2 n-1} P(1 / t)$, giving

$$
P_{j}^{[0,-2 n]}(\mu) \equiv P_{2 n-j-1}^{[0,-2 n]}(\mu) .
$$

In what follows we assume $n$ integer and leave the easy modifications, needed for the half integral case, to the reader.

With the previous notations we have the following:

Lemma 1. Put $Z=\sqrt{ }(2(\cosh v-\cosh u))$ for $v>u>0$. Then for $\lambda$ real

$$
\frac{2^{2 n}}{\pi} \int_{u}^{\infty} \frac{\sin \lambda v d v}{Z\left(2 \cosh \frac{v}{2}+Z\right)^{2 n}}=\frac{1}{2} P_{-1 / 2-n+i \lambda}^{[0,2 n]}(\mu) \tanh \pi \lambda
$$

$$
-\frac{1}{2 \pi i} \sum_{j=0}^{2 n-1} \frac{P_{j}^{[0,-2 n]}(\mu)}{(1+\mu)^{2 n}} \frac{\cosh \left(j-n+\frac{1}{2}-i \lambda\right) u}{j-n+\frac{1}{2}-i \lambda} .
$$

To prove (13) assume first $(n-1)<\operatorname{Re} z<n$, and denote by $I\left(I^{\prime}\right)$ the integral of $G_{n}(t, \mu) t^{n-z-1}$ along the real line from $-\infty$ to $+\infty$, taking the upper (lower resp.) halves of the cuts along $[-\infty, 0]$ and $\left[e^{-u}, e^{u}\right]$. It is easy to see, that $I=I^{\prime}=0$, since the integrand is $O\left(r^{n-R_{\theta}{ }^{-1}}\right)$ for $|t|=r$. Putting $I_{j}\left(I_{j}^{\prime} ; j=1,2,3,4\right)$ for the parts of $I\left(I^{\prime}\right)$ corresponding to $[-\infty, 0],\left[0, e^{-u}\right],\left[e^{-u}, e^{u}\right]$ and $\left[e^{u},+\infty\right]$ resp., one has

$$
I_{1}+I_{1}^{\prime}=\frac{e^{2 \pi i z}+1}{e^{2 \pi i z}-1} \times P_{z-n}^{[0,2 n]}(\mu),
$$


$I_{2}+I_{2}^{\prime}+I_{4}+I_{4}^{\prime}$

$$
=\frac{2 \cdot 2^{2 n}}{i \pi} \int_{u}^{+\infty} \frac{\sinh \left(z+\frac{1}{2}\right) v}{Z\left(2 \cosh \frac{v}{2}+Z\right)^{2 n}} d v-\frac{1}{i \pi} \int_{e^{u}}^{\infty} \frac{P(t)}{t^{2 n+2+1}} d t
$$

and

$$
I_{3}+I_{3}^{\prime}=-\frac{1}{2 \pi i} \int_{e^{-u}}^{e^{u}} \frac{P(t)}{t^{2 n+2+1}} d t
$$

whence through analytic continuation and the substitution $z=-\frac{1}{2}$ $+i \lambda$ we obtain (13).

Next we express the functions $P_{z}^{[0, \pm 2 n]}(\mu)$, occurring in (13), by aid of the functions $\left\{a_{n}^{(\lambda)}(\mu), a_{n}^{(l)}(\mu)\right\}$; the following considerations are valid for all $n$. In order to do this, we give certain realizations of the representations in $T$. We denote the subgroup of $G$, consisting of matrices having 0 in the left lower corner, by $S$ and observe that every element in $G$ has exactly two representations of the form so $\left(s \in S, o \in G_{1}\right)$. For $o \in G_{1}, a \in G$ we denote by $s(o a) \in S$ and $o \bar{a} \in G_{1}$ the elements of one of the pairs, the product of which is $o a$. Putting $\gamma=-e(e=$ unit in $G)$, we write $H_{+}\left(H_{-}\right)$for the subspace of the Hilbert space, with a norm $\|f\|^{2}=\int_{G_{1}}|f(o)|^{2} d \mu(o)(d \mu(o)=d \phi / 4 \pi)$ of functions on $G_{1}$, consisting of elements satisfying $f(\gamma o)=f(o)(f(\gamma o)$ $=-f(o)$ resp. $)$. Then we obtain a realization $U(a)$ of $C_{q}^{(o)}\left(C_{q}^{(1 / 2)}, q>\frac{1}{4}\right)$ in $H_{+}\left(H_{-}\right)$by setting $(U(a) f)(o)=\left|[s(o a)]_{22}\right|^{-1+2 i \lambda} f(o \bar{a}) \quad(U(a) f)(o)$ $=s g\left([s(o a)]_{22}\right)\left|[s(o a)]_{22}\right|-1+i \lambda f(o \bar{a})$ resp., $\lambda=\sqrt{ }\left(q-\frac{1}{4}\right)$. To obtain a realization of $D_{l}^{+}\left(D_{l}^{-}\right)$, we take the Hilbert space $H_{l}^{+}\left(H_{l}^{-}\right)$spanned by the functions $\left\{e^{-i n \phi} ; n \geqq l, n-l=\right.$ integer $\}$ ( $n \leqq-l$ resp.) the norm of $e^{-i n \phi}$ being defined by

$$
\left(\begin{array}{c}
|n|+l-1 \\
|n|-l
\end{array}\right)^{-1 / 2}
$$

for $n \neq m$ the corresponding functions are orthogonal. For sufficiently regular elements of $H_{l}^{+}\left(H_{l}^{-}\right.$resp.) we put $(U(a) f)(o)=\left[s_{22}(o a)\right]^{-2 l} f(o \bar{a})$ and extend by continuity. In each of these cases the part of the system $\left\{e^{-i n \phi}\right\}$ lying in the resp. representation space forms a complete orthonormal system diagonalizing $U\left(g_{\phi}\right)$. Hence we get

$$
a_{n}^{(\lambda)}(\mu)=\left(\frac{1+\mu}{2}\right)^{n} \frac{1}{\pi} \int_{0}^{\pi}\left(\mu+\cos \phi \sqrt{ }\left(\mu^{2}-1\right)\right)^{-1 / 2-n+i \lambda}
$$

$$
\cdot \operatorname{Re}\left[\left(1+\left(\frac{\mu-1}{\mu+1}\right)^{-1 / 2} e^{i \phi}\right)^{2 n}\right] d \phi
$$


and

$$
\begin{aligned}
a_{n}^{(l)}(\mu)= & \left(\frac{1+\mu}{2}\right)^{-n} \frac{1}{\pi} \int_{0}^{\pi}\left(\mu+\cos \phi \sqrt{ }\left(\mu^{2}-1\right)\right)^{n-l} \\
& \cdot \operatorname{Re}\left[\left(1+\left(\frac{\mu-1}{\mu+1}\right)^{1 / 2} e^{i \phi}\right)^{2 n}\right] d \phi .
\end{aligned}
$$

Using the substitution $e^{v}=\mu+\cos \phi \sqrt{ }\left(\mu^{2}-1\right)$ this gives

$$
a_{n}^{(\lambda)}(\mu)=\left(\frac{1+\mu}{2}\right)^{n}\left[\frac{2^{2 n}}{\pi} \int_{0}^{u} \frac{\cos \lambda v}{Z} 2 \operatorname{Re}\left(2 \cosh \frac{v}{2}+i Z\right)^{-2 n} d v\right]
$$

and

$$
a_{n}^{(l)}(\mu)=\left(\frac{1+\mu}{2}\right)^{-n}\left[\frac{1}{2 \pi \cdot 2^{2 n}} \int_{-u}^{u} \frac{e^{-(l-1 / 2) v}}{Z}\right.
$$

$$
\left.\cdot 2 \operatorname{Re}\left(2 \cosh \frac{v}{2}+i Z\right)^{-2 n} d v\right]
$$

But the factor of $((1+\mu) / 2)^{n}\left(((1+\mu) / 2)^{-n}\right)$ in $\left(14^{\prime}\right)$ can be obtained from (10) by putting $z=-\frac{1}{2}-n+i \lambda\left(z=n-l\right.$, and $G_{-n}$ in place of $G_{n}$ resp.) and by letting $C$ be the interval $\left[e^{-u}, e^{u}\right]$ run over twice clockwise. Hence finally

$$
\begin{aligned}
& a_{n}^{(\lambda)}(\mu)=\left(\frac{1+\mu}{2}\right)^{n} P_{-1 / 2-n+i \lambda}^{[0,2 n]}(\mu), \quad(0<l \leqq n, n-l=\text { integer }) . \\
& a_{n}^{(l)}(\mu)=\left(\frac{1+\mu}{2}\right)^{-n} P_{n-l}^{[0,-2 n]}(\mu),
\end{aligned}
$$

Comparing the factor of $((1+\mu) / 2)^{n}\left(((1+\mu) / 2)^{-n}\right.$ resp.) in (14), $\left(14^{\prime}\right)$ and (15), we obtain the analogues of the integral representation of Laplace and Dirichlet-Mehler for Legendre polynomials.

Using (15), (13) can be written as

$$
\begin{aligned}
& \left(\frac{1+\mu}{2}\right)^{n} \frac{2^{2 n}}{\pi} \int_{u}^{\infty} \frac{\sin \lambda v}{Z\left(2 \cosh \frac{v}{2}+Z\right)^{2 n}} d v \\
& \quad=\frac{1}{2}(\tanh \pi \lambda) a_{n}^{(\lambda)}(\mu)-\frac{1}{\pi} \sum_{l=1}^{n} a_{n}^{(l)}(\mu) \operatorname{Im} \frac{\cosh \left[l-\frac{1}{2}-i \lambda\right]}{l-\frac{1}{2}-i \lambda} .
\end{aligned}
$$

Writing $\left(13^{\prime}\right)$ as a Mellin transform we could obtain a formula of the type (9) but we prefer to continue in the following manner. Assuming 
$f(u) \in C_{c}^{\infty}$, we multiply both sides of $\left(13^{\prime}\right)$ with $f(u) \sinh u d u$ and integrate. Applying Fubini's theorem, and integrating partially we obtain on the left hand side:

$$
\frac{1}{\lambda} \int_{0}^{\infty} \cos \lambda v F^{\prime}(v) d v
$$

where

$$
F(v)=\frac{2^{2 n}}{\pi} \int_{0}^{v} \frac{\left(\cosh \frac{u}{2}\right)^{2 n} f(u) \sinh u d u}{Z\left(2 \cosh \frac{v}{2}+Z\right)^{2 n}} .
$$

Observe, that we used $F(0)=0$. Now we have

Lemma 2. For $v>0\left|F^{\prime}(v)-(1 / \pi) f(0)\right|<K v$, with $K$ independent of $v$.

Note, that this is just a verification of (8) $\left(r=e^{v}\right)$; we omit the simple proof. Hence to obtain the final result, it suffices to show, that multiplying the right-hand side of $\left(13^{\prime}\right)$ with $2 \lambda$ and integrating according to $\lambda$ over $[0,+\infty]$, we get the right-hand side of (4). This can be done by elementary computations, using

$$
\frac{1}{2 \pi i} \int_{-i \infty}^{i \infty} \frac{e^{-s u}}{k-s} d s=\left\{\begin{array}{ll}
e^{-k u} & \text { for } k>0, \\
0 & \text { for } k<0
\end{array} \quad(u>0) .\right.
$$

\section{BIBLIOGRAPHY}

1. V. A. Bargmann, Irreducible unitary representations of the Lorentz group, Ann. of Math. (2) 48 (1947), 568-640.

2. J. Dixmier, Representations integrables du groupe de De Sitter, Bull. Soc. Math. France 89 (1961), 9-41.

3. Ph. Frank and R. v. Mises, Die Differential und Integralgleichungen der Mechanik und Physik, Vol. 1, 2. Aufl., Vieweg, Braunschweig.

4. Harish-Chandra, Plancherel formula for the $2 \times 2$ real unimodular group, Proc. Nat. Acad. Sci. U.S.A. 38 (1952), 337-342.

5. G. Szegö, Orthogonal polynomials, Amer. Math. Soc. Colloq. Publ. Vol. 23, Amer. Math. Soc., Providence, R. I., 1959.

University of California, Los Angeles 